\title{
Application of the Adomian decomposition method and Laplace transform method to solving the convection diffusion-dissipation equation
}

\author{
Joseph Bonazebi Yindoula ${ }^{1}$, Pare Youssouf ${ }^{2 *}$, Gabriel Bissanga ${ }^{1}$, Francis Bassono $^{2}$, Blaise Some $^{2}$ \\ ${ }^{1}$ University marien ngouabi of Brazzaville \\ ${ }^{2}$ University of Ouagadougou \\ *Corresponding author E-mail: pareyoussouf@yahoo.fr
}

Copyright $\odot 2014$ Pare Youssouf et al. This is an open access article distributed under the Creative Commons Attribution License, which permits unrestricted use, distribution, and reproduction in any medium, provided the original work is properly cited.

\begin{abstract}
In this paper, the Adomian decomposition method and Laplace transform method are used to construct the solution of a convection-diffusion-dissipation equation.
\end{abstract}

AMS subject classification: 47H14; 34G20; 47J25; 65J15.

Keywords: Adomian decomposition method, Laplace transforms, convection, diffusion, dissipation.

\section{Introduction}

When investigating the pollution of water table, we meet a lot of phenomena, such as convection, diffusion, dissipation etc. These phenomena are governed by mathematical models. Many of them are complex partial differential equations. To study their behavior we often with the simulations based on numeric solutions. In this paper, we examine the convection-diffusion-dissipation equation; we use the Adomian decomposition method and sometimes the Laplace transform method to get the exact solution.

\section{The convection diffusion dissipation equation}

The convection-diffusion-dissipation equation in dimension 1 has the following form:

$\frac{\partial u(x, t)}{\partial t}-\alpha \frac{\partial^{2} u(x, t)}{\partial x^{2}}+v(x) \frac{\partial u(x, t)}{\partial x}+c(x) u(x, t)=f(x, t)$

Where $u(x, t)$ is the concentration of the pollutant, $\alpha$ is a constant, $v(x)$ is the speed of water, $c(x)$ is the dissipation function, $f(x, t)$ is the function source, $x$ is a space variable and $t$ stands for time.

Remark-1:

$-\alpha \frac{\partial^{2} u(x, t)}{\partial x^{2}}$ is the diffusion term, $v(x) \frac{\partial u(x, t)}{\partial x}$ is the convection term, $c(x) u(x, t)$ and is the dissipation term.

\subsection{The convection diffusion dissipation}

Let's consider the following initial and boundary value problem: 
$(A):\left\{\begin{array}{l}\frac{\partial u(x, t)}{\partial t}-\frac{\partial^{2} u(x, t)}{\partial x^{2}}+\frac{\partial u(x, t)}{\partial x}+u(x, t)=f(x, t) \\ u(x, 0)=\cos (x) \\ u(0, t)=\cos (t) \\ u(10, t)=\cos (10) \cos (t)\end{array}\right.$

With

$f(x, t)=-\cos (x) \sin (t)+2 \cos (x) \cos (t)-\sin (x) \cos (t)$

And $(x, t) \in \Omega=[0,10] \times[0, T]$.

We are going to solve problem $(A)$ using the Adomian decomposition method and the Laplace transform method.

\subsubsection{Application of the adomian decomposition method (ADM)}

The general theory of the Adomian decomposition method can be found in [1-10].

From (2) and (3), we have:

$\frac{\partial u(x, t)}{\partial t}=\frac{\partial^{2} u(x, t)}{\partial x^{2}}-\frac{\partial u(x, t)}{\partial x}-u(x, t)-\cos (x) \sin (t)+2 \cos (x) \cos (t)-\sin (x) \cos (t)$

From (4) we have:

$$
\left\{\begin{aligned}
u(x, t)= & u(x, 0)+\int_{0}^{t} \frac{\partial^{2} u(x, s)}{\partial x^{2}} d s-\int_{0}^{t} \frac{\partial u(x, s)}{\partial x} d s-\int_{0}^{t} u(x, s) d s+ \\
& \int_{0}^{t}(-\cos (x) \sin (s)+2 \cos (x) \cos (s)-\sin (x) \cos (s)) d s \\
= & \cos (x) \cos (t)+2 \cos (x) \sin (t)-\sin (x) \sin (t)+ \\
& \int_{0}^{t} \frac{\partial^{2} u(x, s)}{\partial x^{2}} d s-\int_{0}^{t} \frac{\partial u(x, s)}{\partial x} d s-\int_{0}^{t} u(x, s) d s
\end{aligned}\right.
$$

From (2) and (3) we have:

$u(10, t)-u(0, t)-\int_{0}^{10} \frac{\partial^{2} u(z, t)}{\partial x^{2}} d z+\int_{0}^{10} \frac{\partial u(z, t)}{\partial x} d z+\int_{0}^{10} u(z, t) d z+$

$\sin (t) \int_{0}^{10} \cos (z) d z-2 \cos (t) \int_{0}^{10} \cos (z) d z+\cos (t) \int_{0}^{10} \sin (z) d z=0$

(6) $\Leftrightarrow$

$u(10, t)-u(0, t)-\int_{0}^{10} \frac{\partial^{2} u(z, t)}{\partial x^{2}} d z+\int_{0}^{10} \frac{\partial u(z, t)}{\partial x} d z+\int_{0}^{10} u(z, t) d z+$

$\sin (t) \sin (10)-2 \cos (t) \sin (10)+(1-\cos (10)) \cos (t)=0$

(7) $\Leftrightarrow$

$\cos (10) \cos (t)-\cos (t)-\int_{0}^{10} \frac{\partial^{2} u(z, t)}{\partial x^{2}} d z+\int_{0}^{10} \frac{\partial u(z, t)}{\partial x} d z+\int_{0}^{10} u(z, t) d z+$

$\sin (t) \sin (10)-2 \cos (t) \sin (10)+\cos (t)-\cos (t) \cos (10)=0$

(8) $\Leftrightarrow$

$\int_{0}^{10} \frac{\partial^{2} u(z, t)}{\partial x^{2}} d z-\int_{0}^{10} \frac{\partial u(z, t)}{\partial x} d z-\int_{0}^{10} u(z, t) d z-\sin (t) \sin (10)+2 \cos (t) \sin (10)=0$

From (5) and (9), we obtain: 


$$
\left\{\begin{aligned}
u(x, t)= & \cos (x) \cos (t)+2 \cos (x) \sin (t)-\sin (x) \sin (t)- \\
& \sin (t) \sin (10)+2 \cos (t) \sin (10)+ \\
& \int_{0}^{t} \frac{\partial^{2} u(x, s)}{\partial x^{2}} d s-\int_{0}^{t} \frac{\partial u(x, s)}{\partial x} d s-\int_{0}^{t} u(x, s) d s+ \\
& \int_{0}^{10} \frac{\partial^{2} u(z, t)}{\partial x^{2}} d z-\int_{0}^{10} \frac{\partial u(z, t)}{\partial x} d z-\int_{0}^{10} u(z, t) d z
\end{aligned}\right.
$$

According to the ADM, we suppose that the solution of (2) has the following form:

$$
u(x, t)=\sum_{n=0}^{+\infty} u_{n}(x, t)
$$

From (10) and (11), we construct the following Adomian algorithm:

$$
\left\{\begin{aligned}
u_{0}(x, t)= & \cos (x) \cos (t) \\
u_{1}(x, t)= & 2 \cos (x) \sin (t)-\sin (x) \sin (t)-\sin (t) \sin (10)+2 \cos (t) \sin (10)+ \\
& \int_{0}^{t} \frac{\partial^{2} u_{0}(x, s)}{\partial x^{2}} d s-\int_{0}^{t} \frac{\partial u_{0}(x, s)}{\partial x} d s-\int_{0}^{t} u_{0}(x, s) d s+ \\
& \int_{0}^{10} \frac{\partial^{2} u_{0}(z, t)}{\partial x^{2}} d z-\int_{0}^{10} \frac{\partial u_{0}(z, t)}{\partial x} d z-\int_{0}^{10} u_{0}(z, t) d z \\
u_{n+1}(x, t)= & \int_{0}^{t} \frac{\partial^{2} u_{n}(x, s)}{\partial x^{2}} d s-\int_{0}^{t} \frac{\partial u_{n}(x, s)}{\partial x} d s-\int_{0}^{t} u_{n}(x, s) d s+ \\
& \int_{0}^{10} \frac{\partial^{2} u_{n}(z, t)}{\partial x^{2}} d z-\int_{0}^{10} \frac{\partial u_{n}(z, t)}{\partial x} d z-\int_{0}^{10} u_{n}(z, t) d z ; n \geq 1
\end{aligned}\right.
$$

Which gives us:

$u_{1}(x, t)=0 \Rightarrow u_{n}(x, t)=0, \forall n \geq 1$

Thus the solution of (2) is:

$u(x, t)=\cos (x) \cos (t)$

\subsubsection{Application of the Laplace transforms method}

Let's note the Laplace transform by:

$U(x, p)=L_{P}(u(x, t))=\int_{0}^{+\infty} u(x, t) e^{-p t} d t$

Applying the Laplace transform to the equation (2), we obtain:

$p U(x, p)-u(x, 0)-\frac{d^{2} U(x, p)}{d x^{2}}+\frac{d U(x, p)}{d x}+U(x, p)=\frac{-1+2 p}{p^{2}+1} \cos (x)-\frac{p}{p^{2}+1} \sin (x)$

(16) $\Leftrightarrow$

$-\frac{d^{2} U(x, p)}{d x^{2}}+\frac{d U(x, p)}{d x}+(1+p) U(x, p)=\left(1+\frac{-1+2 p}{p^{2}+1}\right) \cos (x)-\frac{p}{p^{2}+1} \sin (x)$

(17) $\Leftrightarrow$

$\frac{d^{2} U(x, p)}{d x^{2}}-\frac{d U(x, p)}{d x}-(1+p) U(x, p)=\frac{p}{p^{2}+1} \sin (x)-\left(\frac{p^{2}+2 p}{p^{2}+1}\right) \cos (x)$

We remark that:

$\left\{\begin{array}{l}U(0, p)=L(u(0, t))=L(\cos (t))=\frac{p}{p^{2}+1} \\ U(10, p)=L(u(10, t))=\cos (10) L(\cos (t))=\frac{p}{p^{2}+1} \cos (10)\end{array}\right.$

The solution of (18) is: 
$U(x, p)=c_{1} e^{\left(\frac{1+\sqrt{4 p+5}}{2}\right) x}+c_{2} e^{-\left(\frac{1+\sqrt{4 p+5}}{2}\right) x}+\frac{p}{p^{2}+1} \cos (x)$

Using the boundary conditions (19), we obtain $c_{1}=c_{2}=0$, and $U(x, p)=\frac{p}{p^{2}+1} \cos (x)$.

Applying the inverse Laplace transform, we obtain:

$u(x, t)=\cos (t) \cos (x)$

\section{Remark-2}

Both methods give us the same result.

a) The convection-diffusion-dissipation equation with variable coefficients

Let's consider the following initial and boundary value problem:

$$
(B):\left\{\begin{array}{l}
\frac{\partial u(x, t)}{\partial t}-\frac{1}{2} \frac{\partial^{2} u(x, t)}{\partial x^{2}}+x(5-x) \cos (x) \frac{\partial u(x, t)}{\partial x}+3 x^{2} u(x, t)=f(x, t) \\
u(x, 0)=\cos (x) \\
u(0, t)=\cos (t) \\
u(10, t)=\cos (10) \cos (t)
\end{array}\right.
$$

With

$f(x, t)=-\cos (x) \sin (t)+\left(0.5+3 x^{2}\right) \cos (x) \cos (t)-x(5-x) \cos (x) \sin (x) \cos (t)$

And $(x, t) \in \Omega=[0,10] \times[0, T]$.

We are going to solve problem $(B)$ using the Adomian decomposition method.

From (22), we have:

$\frac{\partial u(x, t)}{\partial t}=f(x, t)+\frac{1}{2} \frac{\partial^{2} u(x, t)}{\partial x^{2}}-x(5-x) \cos (x) \frac{\partial u(x, t)}{\partial x}-3 x^{2} u(x, t)$

From (24), we have

$$
\left\{\begin{aligned}
u(x, t)= & u(x, 0)+\int_{0}^{t} f(x, s) d s+\frac{1}{2} \int_{0}^{t} \frac{\partial^{2} u(x, s)}{\partial x^{2}} d s- \\
& x(5-x) \cos (x) \int_{0}^{t} \frac{\partial u(x, s)}{\partial x} d s-3 x^{2} \int_{0}^{t} u(x, s) d s \\
= & \cos (x)+\int_{0}^{t}\left(-\cos (x) \sin (s)+\left(0.5+3 x^{2}\right) \cos (x) \cos (s)-x(5-x) \cos (x) \sin (x) \cos (s)\right) d s+ \\
& \frac{1}{2} \int_{0}^{t} \frac{\partial^{2} u(x, s)}{\partial x^{2}} d s-x(5-x) \cos (x) \int_{0}^{t} \frac{\partial u(x, s)}{\partial x} d s-3 x^{2} \int_{0}^{t} u(x, s) d s
\end{aligned}\right.
$$

From (22), we have:

$\int_{0}^{10} \frac{\partial u(x, t)}{\partial t} d x-\frac{1}{2} \int_{0}^{10} \frac{\partial^{2} u(x, t)}{\partial x^{2}} d x+\int_{0}^{10} x(5-x) \cos (x) \frac{\partial u(x, t)}{\partial x} d x+\int_{0}^{10} 3 x^{2} u(x, t) d x-\int_{0}^{10} f(x, t) d x=0$

$(26) \Leftrightarrow$

$\left\{\begin{array}{l}\int_{0}^{10} \frac{\partial u(x, t)}{\partial t} d x-\frac{1}{2} \int_{0}^{10} \frac{\partial^{2} u(x, t)}{\partial x^{2}} d x+\int_{0}^{10} 3 x^{2} u(x, t) d x+\sin (t) \sin (10)- \\ 50 \cos ^{2}(10) \cos (t)-\int_{0}^{10}\left(5 \cos (x)+x^{2} \sin (x)-2 x \cos (x)-5 \sin (x)\right) u(x, t) d x- \\ \left(\frac{1}{2} \sin 10-60 \cos 10+294 \sin 10\right) \cos (t)+\left(\frac{99}{8} \cos 20-\frac{15}{8} \sin 20+\frac{1}{8}\right) \cos (t)=0\end{array}\right.$

From (25) and (27), we obtain: 


$$
\left\{\begin{aligned}
u(x, t)= & \cos (x) \cos (t)+\left(\frac{1}{2}+3 x^{2}\right) \cos (x) \sin (t)-x(5-x) \cos (x) \sin (x) \sin (t)+ \\
& \frac{1}{2} \int_{0}^{t} \frac{\partial^{2} u(x, s)}{\partial x^{2}} d s-\left(5 x-x^{2}\right) \cos (x) \int_{0}^{t} \frac{\partial u(x, s)}{\partial x} d s-3 x^{2} \int_{0}^{t} u(x, s) d s+ \\
& \int_{0}^{10} \frac{\partial u(x, t)}{\partial t} d x-\frac{1}{2} \int_{0}^{10} \frac{\partial^{2} u(x, t)}{\partial x^{2}} d x-\int_{0}^{10} 3 x^{2} u(x, t) d x++\sin (t) \sin (10) \\
- & 50 \cos ^{2}(10) \cos (t)-\int_{0}^{10}\left(5 \cos x+x^{2} \sin x-2 x \cos x-5 x \sin x\right) u(x, t) d x- \\
& \left(\frac{1}{2} \sin 10+60 \cos 10+294 \sin 10\right) \cos (t)+\left(\frac{99}{8} \cos 20-\frac{15}{8} \sin 20+\frac{1}{8}\right) \cos (t)
\end{aligned}\right.
$$

According to the ADM, we suppose that the solution off (22) has the following form:

$$
u(x, t)=\sum_{n=0}^{+\infty} u_{n}(x, t)
$$

From (28) and (29), we construct the following Adomian algorithm:

$$
\left\{\begin{aligned}
u_{0}(x, t)= & \cos (x) \cos (t) \\
u_{1}(x, t)= & \left(\frac{1}{2}+3 x^{2}\right) \cos (x) \sin (t)-x(5-x) \cos (x) \sin (x) \sin (t)+\sin (t) \sin (10)- \\
& 50 \cos ^{2}(10) \cos (t)-\int_{0}^{10}\left(5 \cos x+x^{2} \sin x-2 x \cos x-5 x \sin x\right) u_{0}(x, t) d x- \\
& \left(\frac{1}{2} \sin 10+60 \cos 10+294 \sin 10\right) \cos (t)+\left(\frac{99}{8} \cos 20-\frac{15}{8} \sin 20+\frac{1}{8}\right) \cos (t)+ \\
& \frac{1}{2} \int_{0}^{t} \frac{\partial^{2} u_{0}(x, s)}{\partial x^{2}} d s-\left(5 x-x^{2}\right) \cos (x) \int_{0}^{t} \frac{\partial u_{0}(x, s)}{\partial x} d s-3 x^{2} \int_{0}^{t} u_{0}(x, s) d s+ \\
& \int_{0}^{10} \frac{\partial u_{0}(x, t)}{\partial t} d x-\frac{1}{2} \int_{0}^{10} \frac{\partial^{2} u_{0}(x, t)}{\partial x^{2}} d x-\int_{0}^{10} 3 x^{2} u_{0}(x, t) d x \\
u_{n+1}(x, t)= & \frac{1}{2} \int_{0}^{t} \frac{\partial^{2} u_{n}(x, s)}{\partial x^{2}} d s-\left(5 x-x^{2}\right) \cos (x) \int_{0}^{t} \frac{\partial u_{n}(x, s)}{\partial x} d s-3 x^{2} \int_{0}^{t} u_{n}(x, s) d s+ \\
& \int_{0}^{10} \frac{\partial u_{n}(x, t)}{\partial t} d x-\frac{1}{2} \int_{0}^{10} \frac{\partial^{2} u_{n}(x, t)}{\partial x^{2}} d x-\int_{0}^{10} 3 x^{2} u_{n}(x, t) d x- \\
& \int_{0}^{10}\left(5 \cos x+x^{2} \sin x-2 x \cos x-5 x \sin x\right) u_{n}(x, t) d x ; n \geq 1
\end{aligned}\right.
$$

Which gives us:

$u_{1}(x, t)=0 \Rightarrow u_{n}(x, t)=0, \forall n \geq 1$

Thus the solution of (22) is:

$u(x, t)=\cos (x) \cos (t)$

\section{Conclusion}

Through these examples, we showed again the usefulness of the Adomian decomposition method, in the search of an approximate solution of an equation and this method gave us the exact solution.

\section{References}

[1] K. ABBAOUI, Les fondements de la méthode décompositionnelle d'Adomian et application à la résolution de problèmes issus de la médecine. Thèse de doctorat de l'Université Paris VI. Octobre 1995.

[2] K. ABBAOUI and Y. CHERRUAULT, Convergence of Adomian method applied to differential equations. Math. Comput. Modeling (28, 5), pp103-109, 1994. 
[3] K. ABBAOUI and Y. CHERRUAULT, Convergence of Adomian applied to nonlinear equations. Math. Comput> Modeling (20, 9), pp60-73, 1994.

[4] K. ABBAOUI and Y. CHERRUAULT, The Decomposition method applied to the Cauchy problem. Kybernetes, (28, 1), pp68-74, 1999.

[5] N. NGARHASTA, B.SOME, K.ABBAOUI and Y.CHERRUAULT, New numerical study of Adomian method applied to a diffusion model. Kybernetes, Vol.31, no1, pp61-75, 2002.

[6] M. Hussain and Majid Khan Modified Laplace Decomposition Method, Applied Mathematical Sciences, Vol. 4, 2010, no 36, $1769-1783$.

[7] Pierre BAKI-TANGOU, Gabriel BISSANGA, Application of Adomian method to solving the Duffing-Van Der Pol equation. Communications in Mathematical Analysis. Vol.4, No. 2. pp. 30-40 (2008).

[8] AM Wazwaz, Partial differential equations methods and applications, Netherland Balkema Publisher.2002?

[9] Francis BASSONO, PARE Youssouf, Gabriel Bissanga and Blaise SOME, Application of the Adomian Decomposition method and the perturbation method to solving integral equations, AJMMS, Vol 1, No. 2, July-December 2012, pp. 121- 128.

[10] Francis BASSONO, PARE Youssouf, Gabriel BISSANGA and Blaise SOME, Application of the Adomian Decomposition Method and the Perturbation Method to solving a system of perturbation equations, Far East Journal of Applied Mathematics, Vol 72, No 2, 2012, pp 91-99. 